\title{
Agalsidase Alfa Slows the Decline in Renal Function in Patients with Fabry Disease
}

\author{
Sandro Feriozzi ${ }^{\mathrm{a}}$ Andreas Schwarting ${ }^{\mathrm{b}}$ Gere Sunder-Plassmann ${ }^{\mathrm{d}}$ \\ Michael West ${ }^{\mathrm{e}}$ Markus Cybullac \\ on behalf of the International Fabry Outcome Survey Investigators \\ ${ }^{a}$ Nephrology and Dialysis, Belcolle Hospital, Viterbo, Italy; ${ }^{b}$ Department of Medicine, University Hospital

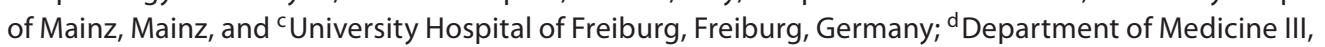

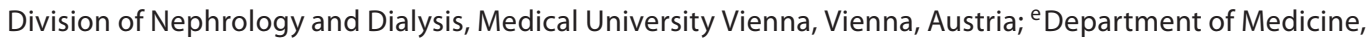 \\ Division of Nephrology, Dalhousie University, Halifax, N.S., Canada
}

\section{Key Words}

Agalsidase alfa • Enzyme replacement therapy •

Fabry disease $\cdot$ Glomerular filtration rate $\cdot$ Renal function

\begin{abstract}
The aim of this study was to determine the effects of enzyme replacement therapy with agalsidase $\alpha$ on renal function in patients with Fabry nephropathy. Serum creatinine data were collected from 165 adult patients during 3 years of treatment. Serum creatinine increased in all men whereas it was stable in women, except in stage II renal disease (Kidney Disease Outcomes Quality Initiative). The estimated glomerular filtration rate (eGFR) declined in males with stage I and II (from $115.0 \pm 22.2$ to $98.3 \pm 27.3$ and from $76.5 \pm 8.1$ to $66.3 \pm 21.6 \mathrm{ml} / \mathrm{min} / 1.73 \mathrm{~m}^{2}$, respectively; both $\mathrm{p}<0.01$ ), whereas eGFR was stable in stage III. In females, eGFR was stable in stages I and III, and decreased in stage II (from 72.5 \pm 8.3 to $67.3 \pm 13.6 \mathrm{ml} / \mathrm{min} / 1.73 \mathrm{~m}^{2} ; \mathrm{p}=0.01$ ). The 24 -hour proteinuria was $<1 \mathrm{~g}$ in all patients, and most patients (96\%) were treated with angiotensin receptor blockers (ARBs) or angiotensin-converting enzyme (ACE) inhibitors. Agalsidase $\alpha$ in combination with ACE inhibitors/ARB may be effective in slowing the deterioration in renal function in Fabry nephropathy.

Copyright $\odot 2008$ S. Karger AG, Basel
\end{abstract}

\section{Introduction}

Fabry disease is an X-linked lysosomal storage disorder caused by complete or partial deficiency in the enzyme $\alpha$-galactosidase $\mathrm{A}$, which results in accumulation of globotriaosylceramide $\left(\mathrm{Gb}_{3}\right)$ within lysosomes in the cells of many tissues, including the kidneys [1]. Renal manifestations (proteinuria and progressive renal impairment) occur relatively early in the course of Fabry disease and, in many patients, progress to renal failure [2].

The disease tends to be more severe and to progress more rapidly in males than in females, with renal failure occurring at approximately 40 years of age in men; however, significant numbers of heterozygous females also have a poor renal prognosis [3].

Enzyme replacement therapy (ERT) has been available since 2001. Studies with agalsidase $\alpha$ and $\beta$ have shown that ERT may significantly slow the rate of renal dysfunction $[4,5]$. ERT was associated with the clearance of microvascular endothelial deposits of $\mathrm{Gb}_{3}$ from the kidneys, heart and skin of patients with Fabry disease and with an improvement in both histological lesions and renal function.

Interpretation of the available data, however, is limited due to the small number of patients, disease heterogene-

\section{KARGER}

Fax +4161306 1234 E-Mail karger@karger.ch www.karger.com
Sandro Feriozzi

Ospedale Belcolle, Nephrology and Dialysis

Strada Sammartinese snc

IT-01100 Viterbo (Italy)

Tel./Fax +39 076151 4703, E-Mail sandro.feriozzi@tiscali.it 
ity and the short duration of initial studies [6]. With regard to renal function, it is important to establish the effect of ERT in relation to the degree of existing renal impairment and the role of concomitant medication. There are also outstanding questions concerning the dosage of ERT and the time of treatment initiation, particularly in pediatric patients and in females with no, or only mild, features of Fabry disease [7].

To be able to study a larger number of patients clinically, the Fabry Outcome Survey (FOS) was initiated in 2001 to monitor the long-term effects and the safety of ERT with agalsidase $\alpha[8]$.

The aim of the present study was to determine the effect of 3 years of ERT with agalsidase $\alpha$ on renal function in a large cohort of patients enrolled in FOS.

\section{Patients and Methods}

FOS is a worldwide database of patients with Fabry disease who are receiving, or are candidates for, ERT with agalsidase $\alpha$ (Replagal ${ }^{\circledR}$; Shire Human Genetic Therapies, Cambridge, Mass., USA). The database and criteria for inclusion have been described elsewhere [8].

Data from adult FOS patients ( $>18$ years of age) with renal assessments (serum creatinine) at the start of ERT and throughout the 3 -year treatment were analyzed. Patients with a renal transplant or on dialysis were excluded. Measurements of serum creatinine recorded in FOS were adjusted according to the Jaffe noncompensated detection method [9]. Creatinine values were used to estimate the glomerular filtration rate (GFR) according to the Modification of Diet in Renal Disease (MDRD) formula [10]. Measurements of serum creatinine and estimated GFR (eGFR) were reported at baseline and after 3 years of ERT for all patients. The increase in serum creatinine and the decrease in eGFR were calculated from the differences between the mean values at baseline and at the follow-up visit at $\geq 3$ years of ERT. The rate of reduction of eGFR per year was also calculated.

In all patients, 24-hour urinary protein excretion was assessed at baseline and annually. Values $>2 \mathrm{~g} / 24 \mathrm{~h}$ were considered as missing values. In addition, all data regarding blood pressure and antihypertensive therapy during the study were collected. Blood pressure was recorded at each clinic visit every 6 months. Due to the global nature of FOS, arterial hypertension was defined by individual physicians according to national and local guidelines, generally a blood pressure $>130 / 90 \mathrm{~mm} \mathrm{Hg}$.

At the time of data collection for this analysis, there were details on 1,356 patients in FOS: 848 were treated with agalsidase $\alpha$, and 733 of them were adults at treatment initiation. Of these adults, 238 received ERT for at least 36 months. A further 29 patients were excluded as they had end-stage renal disease. The number of patients eligible for the study was 165 (115 men and 50 women). Each patient received 40 -min infusions of agalsidase $\alpha$ at a dose of $0.2 \mathrm{mg} / \mathrm{kg}$ every 2 weeks.

To analyze the effects of ERT, patients were subdivided according to sex and baseline renal function: stage I represents a
Table 1. Arterial hypertension in male and female patients with Fabry disease at varying stages of renal disease

\begin{tabular}{lcll}
\hline $\begin{array}{l}\text { Stage of } \\
\text { renal disease }\end{array}$ & $\begin{array}{l}\text { Patients } \\
\mathrm{n}\end{array}$ & $\begin{array}{l}\text { Mean age at } \\
\text { baseline, years }\end{array}$ & $\begin{array}{l}\text { Patients with arte- } \\
\text { rial hypertension, } \mathrm{n}\end{array}$ \\
\hline Men & & & \\
$\quad$ Stage I & 66 & $30 \pm 8$ & 6 \\
$\quad$ Stage II & 32 & $40 \pm 10$ & 9 \\
$\quad$ Stage III & 17 & $42 \pm 7$ & 5 \\
$\quad$ Total & 115 & $37 \pm 8$ & 20 \\
\hline Women & & & \\
$\quad$ Stage I & 6 & $43 \pm 13$ & 0 \\
Stage II & 34 & $46 \pm 14$ & 6 \\
$\quad$ Stage III & 10 & $60 \pm 9$ & 5 \\
$\quad$ Total & 50 & $50 \pm 12$ & 11 \\
\hline
\end{tabular}

Stage I: eGFR $\geq 90 \mathrm{ml} / \mathrm{min} / 1.73 \mathrm{~m}^{2}$; stage II: eGFR 60-89 ml/ $\mathrm{min} / 1.73 \mathrm{~m}^{2}$; stage III, eGFR $\leq 59 \mathrm{ml} / \mathrm{min} / 1.73 \mathrm{~m}^{2}$.

normal GFR ( $\left.>90 \mathrm{ml} / \mathrm{min} / 1.73 \mathrm{~m}^{2}\right)$, stage II represents mild renal failure (GFR $60-89 \mathrm{ml} / \mathrm{min} / 1.73 \mathrm{~m}^{2}$ ) and stage III represents moderate/severe renal failure $\left(\mathrm{GFR} \leq 59 \mathrm{ml} / \mathrm{min} / 1.73 \mathrm{~m}^{2}\right)$.

All patients gave written informed consent for data collection and analysis. Ethical approval was obtained from each patient's local health institution.

Data are reported as means $\pm \mathrm{SD}$. Statistical analyses were carried out using the paired Student's t test or the Wilcoxon's signed rank test, as appropriate. A p value $\leq 0.05$ was considered significant.

\section{Results}

Mean age at baseline was 37.3 years (range, 18.4-68.0 years) for men and 49.6 years (18.4-70.9 years) for women (table 1).

There was a small but statistically significant increase in serum creatinine in men at each stage of kidney disease, and for the male group as a whole $(+0.23 \mathrm{mg} / \mathrm{dl} ; \mathrm{p}<$ 0.01 ; table $2 \mathrm{a}$ ). In women, the increase in serum creatinine was significant only in those with stage II disease $(+0.07 \mathrm{mg} / \mathrm{dl} ; \mathrm{p}=0.02)$; however, the increase was significant for the female group as a whole $(0.08 \mathrm{mg} / \mathrm{dl}$; $\mathrm{p}<$ $0.01)$. Overall, $29(17.6 \%)$ patients had a $\geq 33 \%$ increase in serum creatinine. The increase in serum creatinine was significant in the subgroup of patients with hypertension at baseline and in those with no hypertension: patients with hypertension had slightly higher serum creatinine levels at baseline and after treatment than those with no hypertension. The increase in serum creatinine in hypertensive patients was greater in patients using angiotensin 
Table 2. Change in serum creatinine at baseline and after 3 years of ERT with agalsidase $\alpha$ in patients with Fabry disease

a Listed by gender and presence/absence of hypertension

\begin{tabular}{|c|c|c|c|c|c|c|}
\hline \multirow[t]{2}{*}{ Stage of renal disease } & \multicolumn{2}{|c|}{ Serum creatinine, $\mathrm{mg} / \mathrm{dl}$} & \multirow{2}{*}{$\begin{array}{l}\text { Mean change in } \\
\text { serum creatinine } \\
\text { mg/dl }\end{array}$} & \multirow[t]{2}{*}{$\mathrm{p}$ value } & \multirow{2}{*}{$\begin{array}{l}\text { Increase } \\
\geq 33 \%, \%\end{array}$} & \multirow[t]{2}{*}{$\mathrm{n}$} \\
\hline & baseline & after 3 years of ERT & & & & \\
\hline \multicolumn{7}{|l|}{ Men } \\
\hline Stage I & $0.86 \pm 0.12$ & $1.00 \pm 0.27$ & +0.14 & $<0.01$ & 15.2 & \\
\hline Stage II & $1.15 \pm 0.11$ & $1.42 \pm 0.60$ & +0.28 & $<0.01$ & 25.0 & \\
\hline Stage III & $1.67 \pm 0.25$ & $2.14 \pm 0.99$ & +0.47 & 0.04 & 29.4 & \\
\hline Total & $1.06 \pm 0.32$ & $1.28 \pm 0.66$ & +0.23 & $<0.01$ & 20.0 & \\
\hline \multicolumn{7}{|l|}{ Women } \\
\hline Stage I & $0.67 \pm 0.05$ & $0.72 \pm 0.13$ & +0.04 & 0.50 & 16.7 & \\
\hline Stage II & $0.91 \pm 0.09$ & $0.98 \pm 0.19$ & +0.07 & 0.02 & 8.8 & \\
\hline Stage III & $1.22 \pm 0.24$ & $1.35 \pm 0.46$ & +0.13 & 0.16 & 20.0 & \\
\hline Total & $0.94 \pm 0.21$ & $1.02 \pm 0.31$ & +0.08 & $<0.01$ & 12.0 & \\
\hline \multicolumn{7}{|c|}{ Hypertension at baseline $(\mathrm{n}=31)$} \\
\hline Stage I & $0.92 \pm 0.12$ & $1.40 \pm 0.53$ & +0.48 & 0.08 & & 6 \\
\hline Stage II & $1.02 \pm 0.17$ & $1.24 \pm 0.34$ & +0.22 & $<0.01$ & & 14 \\
\hline Stage III & $1.40 \pm 0.25$ & $1.53 \pm 0.41$ & +0.13 & 0.28 & & 11 \\
\hline Total & $1.13 \pm 0.28$ & $1.37 \pm 0.42$ & +0.24 & $<0.01$ & & \\
\hline \multicolumn{7}{|c|}{ No hypertension at baseline $(\mathrm{n}=134)$} \\
\hline Stage I & $0.84 \pm 0.13$ & $0.93 \pm 0.20$ & +0.10 & $<0.01$ & & 66 \\
\hline Stage II & $1.03 \pm 0.16$ & $1.18 \pm 0.53$ & +0.16 & 0.02 & & 52 \\
\hline Stage III & $1.58 \pm 0.36$ & $2.06 \pm 1.10$ & +0.49 & 0.03 & & 16 \\
\hline Total & $1.00 \pm 0.29$ & $1.17 \pm 0.62$ & +0.17 & $<0.01$ & & \\
\hline
\end{tabular}

b Listed by use or non-use of ACE inhibitors or ARBs

\begin{tabular}{|c|c|c|c|c|}
\hline \multirow[t]{2}{*}{ Stage of renal disease } & \multicolumn{2}{|c|}{ Serum creatinine, $\mathrm{mg} / \mathrm{dl}$} & \multirow{2}{*}{$\begin{array}{l}\text { Mean change in } \\
\text { serum creatinine } \\
\mathrm{mg} / \mathrm{dl}\end{array}$} & \multirow[t]{2}{*}{$\mathrm{n}$} \\
\hline & baseline & after 3 years of ERT & & \\
\hline \multicolumn{5}{|c|}{ Patients not using ARBs or ACE inhibitors $(n=6)$} \\
\hline Stage I & $1.01 \pm 0.05$ & $1.02 \pm 0.19$ & +0.01 & 2 \\
\hline Stage II & 0.86 & 0.99 & +0.13 & 1 \\
\hline Stage III & $1.41 \pm 0.29$ & $1.39 \pm 0.71$ & -0.02 & 3 \\
\hline Total & $1.18 \pm 0.31$ & $1.20 \pm 0.50$ & +0.02 & \\
\hline \multicolumn{5}{|c|}{ Patients using ARBs or ACE inhibitors $(\mathrm{n}=25)$} \\
\hline Stage I & $0.88 \pm 0.13$ & $1.59 \pm 0.56$ & +0.72 & 4 \\
\hline Stage II & $1.03 \pm 0.17$ & $1.26 \pm 0.35$ & +0.23 & 13 \\
\hline Stage III & $1.39 \pm 0.26$ & $1.58 \pm 0.30$ & +0.19 & 8 \\
\hline Total & $1.12 \pm 0.27$ & $1.41 \pm 0.39$ & +0.29 & \\
\hline
\end{tabular}

Stage I: eGFR $\geq 90 \mathrm{ml} / \mathrm{min} / 1.73 \mathrm{~m}^{2}$; stage II: eGFR $60-89 \mathrm{ml} / \mathrm{min} / 1.73 \mathrm{~m}^{2}$; stage III: eGFR $\leq 59 \mathrm{ml} / \mathrm{min} / 1.73 \mathrm{~m}{ }^{2}$.

receptor blockers (ARBs) or angiotensin-converting enzyme (ACE) inhibitors $(+0.29 \mathrm{mg} / \mathrm{dl})$ than in those not using ARBS or ACE inhibitors (+0.02 $\mathrm{mg} / \mathrm{dl}$; table $2 \mathrm{~b}$ ), but the small number of patients involved renders statistical analysis unrealistic.

Enzyme Replacement and Renal Function in Fabry Disease
The eGFR remained stable in men with stage III disease (table 3a). In those with stage I or II renal disease, there was a small but significant decrease in eGFR $(\mathrm{p}<$ 0.01 ). The mean decrease in eGFR in all male patients was $2.66 \pm 5.07 \mathrm{ml} / \mathrm{min} / 1.73 \mathrm{~m}^{2} /$ year, with a mean change of

Am J Nephrol 2009;29:353-361 
Table 3. eGFR (means \pm SD) in patients with Fabry disease at baseline and after 3 years of ERT with agalsidase $\alpha$ a Listed by gender and presence/absence of hypertension

\begin{tabular}{|c|c|c|c|c|c|c|}
\hline \multirow[t]{2}{*}{ Stage of renal disease } & \multicolumn{2}{|c|}{$\mathrm{eGFR}, \mathrm{ml} / \mathrm{min} / 1.73 \mathrm{~m}^{2}$} & \multirow{2}{*}{$\begin{array}{l}\text { Mean change } \\
\text { in eGFR } \\
\mathrm{ml} / \mathrm{min} / 1.73 \mathrm{~m}^{2}\end{array}$} & \multirow{2}{*}{$\begin{array}{l}\mathrm{p} \\
\text { value }\end{array}$} & \multirow{2}{*}{\multicolumn{2}{|c|}{$\begin{array}{l}\text { Slope change } \\
\mathrm{ml} / \mathrm{min} / 1.73 \mathrm{~m}^{2} \\
\text { per year }\end{array}$}} \\
\hline & baseline & after 3 years of ERT & & & & \\
\hline \multicolumn{7}{|l|}{ Men } \\
\hline Stage I & $115.0 \pm 22.2$ & $98.3 \pm 27.3$ & -16.3 & $<0.01$ & -3.19 & \\
\hline Stage II & $76.5 \pm 8.1$ & $66.3 \pm 21.6$ & -10.1 & $<0.01$ & -2.30 & \\
\hline Stage III & $49.1 \pm 6.6$ & $42.8 \pm 19.9$ & -6.3 & 0.18 & -1.29 & \\
\hline Total & $94.5 \pm 30.8$ & $81.2 \pm 32.5$ & -13.3 & $<0.01$ & -2.66 & \\
\hline \multicolumn{7}{|l|}{ Women } \\
\hline Stage I & $103.2 \pm 10.4$ & $96.4 \pm 17.8$ & -6.8 & 0.46 & -1.48 & \\
\hline Stage II & $72.5 \pm 8.3$ & $67.3 \pm 13.6$ & -5.2 & 0.01 & -1.29 & \\
\hline Stage III & $49.5 \pm 8.3$ & $46.3 \pm 13.8$ & -3.2 & 0.28 & -0.74 & \\
\hline Total & $71.6 \pm 17.1$ & $66.6 \pm 19.6$ & -5.0 & $<0.01$ & -1.20 & \\
\hline \multicolumn{7}{|c|}{ Hypertension at baseline $(n=31)$} \\
\hline Stage I $(90+)$ & $102.8 \pm 17.8$ & $69.3 \pm 28.5$ & -33.6 & 0.04 & -5.86 & \\
\hline Stage II (60-90) & $76.1 \pm 8.4$ & $62.5 \pm 16.2$ & -13.5 & $<0.01$ & -3.38 & \\
\hline Stage III $(0-60)$ & $50.9 \pm 4.3$ & $48.4 \pm 18.8$ & -2.5 & 0.68 & -0.41 & \\
\hline Total & $72.3 \pm 21.2$ & $58.8 \pm 20.8$ & -13.5 & $<0.01$ & -2.81 & \\
\hline \multicolumn{7}{|c|}{ No hypertension at baseline $(\mathrm{n}=135)$} \\
\hline Stage I $(90+)$ & $115.0 \pm 21.8$ & $100.8 \pm 24.9$ & -14.2 & $<0.01$ & -2.79 & \\
\hline Stage II (60-90) & $74.0 \pm 8.4$ & $68.0 \pm 18.1$ & -6.1 & $<0.01$ & -1.35 & \\
\hline Stage III $(0-60)$ & $48.2 \pm 8.6$ & $41.2 \pm 16.8$ & -7.0 & 0.05 & -1.54 & \\
\hline Total & $91.1 \pm 29.8$ & $81.0 \pm 30.2$ & -10.2 & $<0.01$ & -2.08 & \\
\hline \multicolumn{7}{|c|}{ b Listed by use or non-use of ACE inhibitors or ARBs } \\
\hline \multirow[t]{2}{*}{ Stage of renal disease } & \multicolumn{2}{|c|}{$\mathrm{eGFR}, \mathrm{ml} / \mathrm{min} / 1.73 \mathrm{~m}^{2}$} & \multirow{2}{*}{$\begin{array}{l}\text { Mean change in } \\
\text { eGFR } \\
\mathrm{ml} / \mathrm{min} / 1.73 \mathrm{~m}^{2}\end{array}$} & \multirow{2}{*}{\multicolumn{2}{|c|}{$\begin{array}{l}\text { Slope change } \\
\mathrm{ml} / \mathrm{min} / 1.73 \mathrm{~m}^{2} \\
\text { per year }\end{array}$}} & $\mathrm{n}$ \\
\hline & baseline & after 3 years of ERT & & & & \\
\hline \multicolumn{7}{|c|}{ Patients not using ARBs or ACE inhibitors $(n=6)$} \\
\hline Stage I & $91.5 \pm 1.4$ & $90.5 \pm 24.4$ & -1.02 & \multicolumn{2}{|c|}{$-1.06 \pm 6.07$} & 2 \\
\hline Stage II & 81.1 & 67.1 & -14.0 & \multirow{2}{*}{\multicolumn{2}{|c|}{$3.35 \pm 11.84$}} & 1 \\
\hline Stage III & $53.4 \pm 4.9$ & $63.2 \pm 34.0$ & +9.82 & & & 3 \\
\hline Total & $70.7 \pm 19.6$ & $73.0 \pm 27.7$ & +2.23 & \multicolumn{2}{|c|}{$0.77 \pm 8.50$} & \\
\hline \multicolumn{7}{|c|}{ Patients using ARBs or ACE inhibitors $(\mathrm{n}=25)$} \\
\hline Stage I & $108.4 \pm 20.0$ & $58.6 \pm 26.5$ & -49.8 & \multicolumn{2}{|c|}{$-8.26 \pm 3.50$} & 4 \\
\hline Stage II & $75.7 \pm 8.6$ & $62.2 \pm 16.8$ & -13.5 & \multicolumn{2}{|c|}{$-3.38 \pm 2.96$} & 13 \\
\hline Stage III & $49.9 \pm 3.9$ & $42.8 \pm 6.4$ & -7.2 & \multicolumn{2}{|c|}{$-1.83 \pm 1.64$} & 8 \\
\hline Total & $72.7 \pm 22.0$ & $55.4 \pm 17.9$ & -17.3 & \multicolumn{3}{|c|}{$-3.66 \pm 3.37$} \\
\hline
\end{tabular}

$-13.3 \mathrm{ml} / \mathrm{min} / 1.73 \mathrm{~m}^{2}(\mathrm{p}<0.01)$ over 3 years. The eGFR in women with stage I or stage III renal disease remained stable. The group with mild renal failure (stage II) had a small but significant change in mean eGFR of $-5.2 \mathrm{ml} /$ $\mathrm{min} / 1.73 \mathrm{~m}^{2}(\mathrm{p}=0.01)$. The mean rate of eGFR decline in all women was $1.20 \pm 3.28 \mathrm{ml} / \mathrm{min} / 1.73 \mathrm{~m}^{2} /$ year, with a mean reduction in eGFR over 3 years of $5.0 \mathrm{ml} / \mathrm{min} / 1.73$ $\mathrm{m}^{2}(\mathrm{p}<0.01)$. For all patients, the rate of decline in eGFR was $2.22 \pm 4.64 \mathrm{ml} / \mathrm{min} / 1.73 \mathrm{~m}^{2} /$ year. The decrease in eGFR was significant in the subgroup of patients with hypertension at baseline and in those with no hypertension, and the mean eGFR decline was similar in the two subgroups of patients $(-2.81 \pm 4.93$ and $-2.08 \pm 4.58$ $\mathrm{ml} / \mathrm{min} / 1.73 \mathrm{~m}^{2} /$ year, respectively). Patients not using 
Table 3 (continued)

c Listed by baseline proteinuria

\begin{tabular}{|c|c|c|c|c|c|}
\hline \multirow[t]{2}{*}{ Stage of renal disease } & \multicolumn{2}{|c|}{$\mathrm{eGFR}, \mathrm{ml} / \mathrm{min} / 1.73 \mathrm{~m}^{2}$} & \multirow{2}{*}{$\begin{array}{l}\text { Mean change } \\
\text { in eGFR ml/ } \\
\mathrm{min} / 1.73 \mathrm{~m}^{2}\end{array}$} & \multirow{2}{*}{$\begin{array}{l}\text { Slope change } \\
\mathrm{ml} / \mathrm{min} / 1.73 \mathrm{~m}^{2} \\
\text { per year }\end{array}$} & \multirow[t]{2}{*}{$\mathrm{n}$} \\
\hline & baseline & after 3 years of ERT & & & \\
\hline \multicolumn{6}{|c|}{ Protein $>500 \mathrm{mg} / 24 \mathrm{~h}$ at baseline $(\mathrm{n}=14)$} \\
\hline Stage I & $112.42 \pm 14.60$ & $85.10 \pm 20.83$ & -27.32 & $-7.08 \pm 6.32$ & 8 \\
\hline Stage II & $69.97 \pm 5.00$ & $57.37 \pm 8.67$ & -12.59 & $-3.08 \pm 2.65$ & 5 \\
\hline Stage III & 49.25 & 100.79 & & & 1 \\
\hline Total & $92.75 \pm 26.57$ & $76.32 \pm 22.10$ & -16.43 & $-3.98 \pm 7.86$ & \\
\hline \multicolumn{3}{|c|}{ Protein $<500 \mathrm{mg} / 24 \mathrm{~h}$ at baseline $(\mathrm{n}=40)$} & & & \\
\hline Stage I & $109.58 \pm 21.74$ & $97.17 \pm 22.00$ & -2.10 & $-2.98 \pm 4.86$ & 16 \\
\hline Stage II & $76.35 \pm 9.87$ & $72.14 \pm 12.42$ & -4.21 & $-0.97 \pm 2.20$ & 17 \\
\hline Stage III & $51.94 \pm 3.52$ & $49.84 \pm 8.16$ & -2.10 & $-0.44 \pm 1.99$ & 7 \\
\hline Total & $85.37 \pm 26.46$ & $78.25 \pm 23.82$ & -7.12 & $-1.68 \pm 3.59$ & \\
\hline
\end{tabular}

Stage I: eGFR $\geq 90 \mathrm{ml} / \mathrm{min} / 1.73 \mathrm{~m}^{2}$; stage II: eGFR $60-89 \mathrm{ml} / \mathrm{min} / 1.73 \mathrm{~m}^{2}$; stage III: eGFR $\leq 59 \mathrm{ml} / \mathrm{min} / 1.73 \mathrm{~m}{ }^{2}$.

ARBs or ACE inhibitors had a small increase in eGFR $\left(+0.77 \pm 8.50 \mathrm{ml} / \mathrm{min} / 1.73 \mathrm{~m}^{2} /\right.$ year; table $\left.3 \mathrm{~b}\right)$, but with only 6 patients not taking ARBs or ACE inhibitors, the significance of this result compared with declines in eGFR in other patients is not clear. The decline in eGFR was numerically larger in patients with urine protein levels $>500 \mathrm{mg} / 24 \mathrm{~h}\left(-3.98 \pm 7.86 \mathrm{ml} / \mathrm{min} / 1.73 \mathrm{~m}^{2} /\right.$ year $)$ than in patients with urine protein levels $<500 \mathrm{mg} / 24 \mathrm{~h}$ $\left(-1.68 \pm 3.59 \mathrm{ml} / \mathrm{min} / 1.73 \mathrm{~m}^{2} /\right.$ year; table $\left.3 \mathrm{c}\right)$.

Hypertension was reported in 31 of the 165 patients at baseline and was present in all groups of patients except women with normal renal function (table 1). At baseline, the prevalence of hypertension was $22 \%$ in women $(11 / 50)$ and $17 \%$ in men (20/115). Mean diastolic blood pressure was $<90 \mathrm{~mm} \mathrm{Hg}$ in all groups at all times. After 3 years of ERT, male patients with stage II and III renal disease and female patients with stage III renal disease showed a non-significant reduction in diastolic blood pressure. Mean systolic blood pressure was $>130 \mathrm{~mm} \mathrm{Hg}$ in males (stages II and III) at baseline and was reduced to normal by the 3rd year of ERT. This reduction was statistically significant in patients with stage II renal disease $(\mathrm{p}<$ 0.01 ). In women, only patients with stage III renal disease had a mean systolic blood pressure $>130 \mathrm{~mm} \mathrm{Hg}$ at baseline. In this group, there was an improvement in blood pressure control after 3 years of ERT, but the mean remained above normal. Patients in stages I and II had normal systolic blood pressure values throughout the study.

Enzyme Replacement and Renal Function in Fabry Disease
Except for women with stage I or III renal disease, all groups had mild proteinuria (between 300 and 1,000 $\mathrm{mg} / 24 \mathrm{~h}$; table $4 \mathrm{a}$ ). The values for proteinuria were characterized by a wide standard deviation in all patients. There was no significant change in proteinuria after 3 years of ERT. Similarly, when data were analyzed for patients with and without hypertension at baseline, there was no obvious difference in proteinuria between subgroups and no significant change after 3 years of ERT. The use or non-use of ARBs or ACE inhibitors had no obvious effect on changes in proteinuria after 3 years of ERT, but data were available for only a small number of patients (table $4 \mathrm{~b}$ ).

\section{Discussion}

Fabry nephropathy is progressive and eventually leads to end-stage renal disease in all male patients and in a smaller number of female patients. Although the shortterm results of ERT are promising, data on long-term effects are scarce.

The results of our study suggest that ERT with agalsidase $\alpha$ slows the decline in renal function in patients with Fabry disease, and that this occurs in both males and females, even in those with advanced renal failure (eGFR $\leq 59 \mathrm{ml} / \mathrm{min} / 1.73 \mathrm{~m}^{2}$ ).

Several studies have investigated the potential benefit of ERT in patients with Fabry disease. Different param-

Am J Nephrol 2009;29:353-361 
Table 4. Proteinuria at baseline and after 3 years of ERT with agalsidase $\alpha$ in patients with Fabry disease

a Listed by gender and presence/absence of hypertension

\begin{tabular}{|c|c|c|c|c|c|}
\hline \multirow[t]{2}{*}{ Stage of renal disease } & \multicolumn{2}{|c|}{ Proteinuria, mg/24 h } & \multirow{2}{*}{$\begin{array}{l}\text { Mean change in } \\
\text { proteinuria, } \mathrm{mg} / 24 \mathrm{~h}\end{array}$} & \multirow[t]{2}{*}{$\mathrm{p}$ value } & \multirow[t]{2}{*}{$\mathrm{n}$} \\
\hline & baseline & after 3 years of ERT & & & \\
\hline \multicolumn{6}{|l|}{ Men } \\
\hline Stage I & $398.8 \pm 435.9$ & $471.4 \pm 347.0$ & $+72.6 \pm 373$ & 0.35 & 24 \\
\hline Stage II & $442.8 \pm 440.5$ & $445.0 \pm 584.2$ & $+2.2 \pm 689$ & 0.99 & 8 \\
\hline Stage III & $476.0 \pm 286.9$ & $654.0 \pm 99.1$ & $+178.0 \pm 335$ & 0.45 & 3 \\
\hline Total & $415.4 \pm 417.1$ & $481.0 \pm 394.1$ & $65 \pm 448$ & 0.39 & \\
\hline \multicolumn{6}{|l|}{ Women } \\
\hline Stage I & $195.0 \pm 49.5$ & $145.0 \pm 63.6$ & $-50.0 \pm 113$ & 0.64 & 2 \\
\hline Stage II & $346.6 \pm 383.9$ & $307.5 \pm 296.7$ & $-39.1 \pm 361$ & 0.68 & 15 \\
\hline Stage III & $246.4 \pm 86.9$ & $336.6 \pm 225.1$ & $+90.2 \pm 223$ & 0.42 & 5 \\
\hline Total & $310.0 \pm 320.9$ & $299.3 \pm 266.8$ & $-10.7 \pm 317$ & 0.88 & \\
\hline \multicolumn{6}{|c|}{ Hypertension at baseline $(\mathrm{n}=12)$} \\
\hline Stage I & 890.0 & 690.0 & & & 1 \\
\hline Stage II & $410.3 \pm 250.1$ & $475.9 \pm 618.7$ & $65.6 \pm 649.4$ & 0.80 & 7 \\
\hline Stage III & $335.5 \pm 297.9$ & $397.0 \pm 216.6$ & $61.5 \pm 194.1$ & 0.57 & 4 \\
\hline Total & $425.3 \pm 284.6$ & $467.4 \pm 477.5$ & $42.1 \pm 496.1$ & 0.78 & \\
\hline \multicolumn{6}{|c|}{ No hypertension at baseline $(\mathrm{n}=45)$} \\
\hline Stage I & $362.8 \pm 417.4$ & $436.6 \pm 348.1$ & $73.8 \pm 363.2$ & 0.32 & 25 \\
\hline Stage II & $366.8 \pm 453.5$ & $302.6 \pm 291.7$ & $-64.2 \pm 412.5$ & 0.54 & 16 \\
\hline Stage III & $329.5 \pm 95.5$ & $514.3 \pm 284.6$ & $184.8 \pm 313.2$ & 0.32 & 4 \\
\hline Total & $361.3 \pm 407.3$ & $395.8 \pm 325.6$ & $34.6 \pm 378.3$ & 0.54 & \\
\hline
\end{tabular}

b Listed by use or non-use of ACE inhibitors or ARBs

\begin{tabular}{|c|c|c|c|c|}
\hline \multirow[t]{2}{*}{ Stage of renal disease } & \multicolumn{2}{|c|}{ Proteinuria, $\mathrm{mg} / 24 \mathrm{~h}$} & \multirow{2}{*}{$\begin{array}{l}\text { Mean change in } \\
\text { proteinuria, } \mathrm{mg} / 24 \mathrm{~h}\end{array}$} & \multirow[t]{2}{*}{$\mathrm{n}$} \\
\hline & baseline & after 3 years of ERT & & \\
\hline \multicolumn{5}{|c|}{ Patients not using ARBs or ACE inhibitors $(\mathrm{n}=4)$} \\
\hline Stage I & 890.0 & 690.0 & & 1 \\
\hline Stage II & 180.0 & 211.0 & & 1 \\
\hline Stage III & $470.0 \pm 438.4$ & $573.0 \pm 123.0$ & $103.0 \pm 315.4$ & 2 \\
\hline Total & $502.5 \pm 386.6$ & $511.8 \pm 219.7$ & $9.3 \pm 231.9$ & \\
\hline \multicolumn{5}{|c|}{ Patients using ARBs or ACE inhibitors $(\mathrm{n}=8)$} \\
\hline Stage I & & & & 6 \\
\hline Stage II & $448.7 \pm 250.3$ & $520.0 \pm 665.6$ & $71.3 \pm 711.2$ & 2 \\
\hline Stage III & $201.0 \pm 41.0$ & $221.0 \pm 41.0$ & $20.0 \pm 82.0$ & \\
\hline Total & $386.8 \pm 241.1$ & $445.3 \pm 579.5$ & $58.5 \pm 602.3$ & \\
\hline
\end{tabular}

Stage I: eGFR $\geq 90 \mathrm{ml} / \mathrm{min} / 1.73 \mathrm{~m}^{2}$; stage II: eGFR $60-89 \mathrm{ml} / \mathrm{min} / 1.73 \mathrm{~m}^{2}$; stage III: eGFR $\leq 59 \mathrm{ml} / \mathrm{min} / 1.73 \mathrm{~m}^{2}$.

eters have been analyzed, including the reduction in $\mathrm{Gb}_{3}$ deposits in the kidneys, heart and skin, the reduction in urinary excretion of $\mathrm{Gb}_{3}$ and changes in renal function from baseline $[4,5,11,12]$. These studies, however, involved small groups of patients for short periods of time. Other studies have employed a different approach.
Schwarting et al. [13], for example, described a negative association of agalsidase $\alpha$ ERT with serum creatinine and duration of ERT in a multivariate analysis; moreover, ERT was able to prevent the progression of renal dysfunction. Banikazemi et al. [14] performed a prospective randomized placebo-controlled study of agalsidase $\beta$ with 
Table 5. Rate of change in GFR in patients with Fabry disease

\begin{tabular}{|c|c|c|c|c|c|c|}
\hline \multirow{2}{*}{$\begin{array}{l}\text { Study and type of } \\
\text { ERT used }\end{array}$} & \multirow{2}{*}{$\begin{array}{l}\text { Patients } \\
\mathrm{n}\end{array}$} & \multirow{2}{*}{$\begin{array}{l}\text { Patient groups } \\
\text { studied }\end{array}$} & \multicolumn{2}{|c|}{$\mathrm{GFR}, \mathrm{ml} / \mathrm{min} / 1.73 \mathrm{~m}^{2}$} & \multirow{2}{*}{$\begin{array}{l}\text { ERT } \\
\text { duration } \\
\text { years }\end{array}$} & \multirow{2}{*}{$\begin{array}{l}\text { Rate of change in } \\
\text { GFR } \mathrm{ml} / \mathrm{min} / 1.73 \mathrm{~m}^{2} \\
\text { per year }\end{array}$} \\
\hline & & & before ERT & after ERT & & \\
\hline Branton et al. [2] & 14 & no ERT & & & 0 & $-12.2 \mathrm{r}$ \\
\hline $\begin{array}{l}\text { De Schoenmakere et al. [15] } \\
\text { (Agalsidase } \beta \text { ) }\end{array}$ & 1 & case report & & & $\begin{array}{l}0 \\
1.5 \\
\end{array}$ & $\begin{array}{l}-6.4 \mathrm{r} \\
-2.2 \mathrm{r}\end{array}$ \\
\hline $\begin{array}{l}\text { Germain et al. [16] } \\
\text { (Agalsidase } \beta \text { ) }\end{array}$ & $\begin{array}{r}52 \\
10 \\
42 \\
8 \\
32 \\
\end{array}$ & $\begin{array}{l}\text { all patients } \\
\text { Prot }>1 \mathrm{~g} / 24 \mathrm{~h} \\
\text { Prot }<1 \mathrm{~g} / 24 \mathrm{~h} \\
\text { GS }>50 \% \\
\text { GS }<50 \%\end{array}$ & $\begin{array}{l}\text { NR } \\
\text { NR } \\
\text { NR } \\
\text { NR } \\
\text { NR }\end{array}$ & $\begin{array}{l}\text { NR } \\
\text { NR } \\
\text { NR } \\
\text { NR } \\
\text { NR } \\
\end{array}$ & $\begin{array}{l}4.5 \\
4.5 \\
4.5 \\
4.5 \\
4.5 \\
\end{array}$ & $\begin{array}{l}-0.4 \mathrm{r} \\
-7.4 \mathrm{r} \\
-1 \mathrm{r} \\
-8.9 \mathrm{r} \\
-1.4 \mathrm{r}\end{array}$ \\
\hline $\begin{array}{l}\text { Schiffmann et al. [17] } \\
\text { (Agalsidase } \alpha \text { ) }\end{array}$ & $\begin{array}{r}24 \\
12 \\
8 \\
4 \\
\end{array}$ & $\begin{array}{l}\text { all patients } \\
\text { stage I } \\
\text { stage II } \\
\text { stage III }\end{array}$ & $\begin{array}{c}88.4 \pm 26 \\
108.7 \pm 14.1 \\
78.6 \pm 8.2 \\
47.1 \pm 9.4 \\
\end{array}$ & $\begin{array}{c}71.5 \pm 32.7 \\
101.5 \pm 12.4 \\
67.1 \pm 17 \\
25.1 \pm 16.4 \\
\end{array}$ & $\begin{array}{l}4 \\
4 \\
4 \\
4\end{array}$ & $\begin{array}{l}-3.3 \mathrm{e} \\
-1.8 \mathrm{e} \\
-2.8 \mathrm{e} \\
-5.5 \mathrm{e}\end{array}$ \\
\hline $\begin{array}{l}\text { Breunig et al. [7] } \\
\text { (Agalsidase } \beta \text { ) }\end{array}$ & $\begin{array}{r}9 \\
16 \\
6\end{array}$ & $\begin{array}{l}\text { GFR }>90 \\
\text { GFR }<90 \\
\text { females }\end{array}$ & $\begin{array}{r}115 \pm 18 \\
71 \pm 17 \\
108 \pm 30\end{array}$ & $\begin{array}{r}102 \pm 14 \\
60 \pm 23 \\
94 \pm 30\end{array}$ & $\begin{array}{l}2 \\
2 \\
2\end{array}$ & $\begin{array}{l}-6.5 \mathrm{e} \\
-5.5 \mathrm{e} \\
-7 \mathrm{e}\end{array}$ \\
\hline $\begin{array}{l}\text { Schiffmann et al. [18] } \\
\text { (Agalsidase } \alpha \text { ) }\end{array}$ & $\begin{array}{l}11 \\
11\end{array}$ & $\begin{array}{l}\text { ERT/2 weeks } \\
\text { ERT/weekly }\end{array}$ & $\begin{array}{l}77.8 \pm 30 \\
51.3 \pm 22.7 \\
\end{array}$ & $\begin{array}{l}53.7 \pm 21 \\
44.7 \pm 24.3\end{array}$ & $\begin{array}{l}3.4 \\
2 \\
\end{array}$ & $\begin{array}{l}-8 \mathrm{r} \\
-3.3 \mathrm{r}\end{array}$ \\
\hline $\begin{array}{l}\text { Tahir et al. [27] } \\
\text { (Agalsidase } \beta \text { ) }\end{array}$ & $\begin{array}{l}4 \\
6\end{array}$ & $\begin{array}{l}\text { GFR }>60 \\
\text { GFR }<60\end{array}$ & $\begin{array}{l}96.9 \pm 17.2 \\
39.4 \pm 10.9\end{array}$ & $\begin{array}{l}\text { ND } \\
\text { ND }\end{array}$ & $\begin{array}{l}4 \\
2.2\end{array}$ & $\begin{array}{l}-1.1 \mathrm{r} \\
-0.23 \mathrm{r}\end{array}$ \\
\hline
\end{tabular}

$\mathrm{e}=$ Mean value of the slope calculated from reported data in the paper; GS = glomerulosclerosis; ND = not done; NR = not reported; Prot $=24$-hour urinary protein excretion; $r=$ mean value of the slope reported in the paper.

heart disease, renal failure and stroke as clinical endpoints. They found a benefit of ERT only in patients with GFR $>55 \mathrm{ml} / \mathrm{min}$ and mild proteinuria.

Changes in renal function over time can best be described by the estimated or measured rate of reduction in GFR per year. In 2002, Branton et al. [2] reported a reduction of $12.2 \mathrm{ml} / \mathrm{min} /$ year in a small group of males with Fabry disease who were not receiving treatment. Since the advent of ERT, the rate of deterioration of GFR per year has been studied either explicitly or data have been provided from which calculations could be made (table 5). In 2003, De Schoenmakere et al. [15] reported a change in GFR in a young female from $-6.4 \mathrm{ml} / \mathrm{min} /$ year without therapy to $-2.2 \mathrm{ml} / \mathrm{min} /$ year with agalsidase $\beta$ and irbesartan. Germain et al. [16] reported a mean change in GFR of $-0.4 \mathrm{ml} / \mathrm{min} /$ year in 52 patients, excluding those with severe progression of renal disease. In addition, when they subdivided patients according to the degree of proteinuria and the percentage of sclerotic glomeruli on renal biopsy, the decreases in GFR were -7.4 $\mathrm{ml} / \mathrm{min} /$ year (proteinuria $>1 \mathrm{~g} / 24 \mathrm{~h}$ ) and $-1.0 \mathrm{ml} / \mathrm{min} /$ year (proteinuria $<1 \mathrm{~g} / 24 \mathrm{~h}$ ), and $-1.4 \mathrm{ml} / \mathrm{min} /$ year $(<50 \%$ sclerotic glomeruli) and $-8.9 \mathrm{ml} / \mathrm{min} /$ year ( $>50 \%$ sclerotic glomeruli). It is therefore possible to calculate the mean eGFR reduction in these groups as -2.2 and -2.9 $\mathrm{ml} / \mathrm{min} /$ year according to the degree of proteinuria and sclerotic glomeruli, respectively. In 25 adults treated for 54 months with agalsidase $\alpha$, Schiffmann et al. [17] revealed a reduction in proteinuria of $1.8 \mathrm{ml} / \mathrm{min} /$ year in stage I, $2.8 \mathrm{ml} / \mathrm{min} /$ year in stage II and $5.5 \mathrm{ml} / \mathrm{min} /$ year in stage III renal disease. Recently, they [18] reported that ERT infused every week rather than every 2 weeks can slow the decline in renal function (to $-3.3 \mathrm{ml} / \mathrm{min} /$ year) in patients with a severe decrease in GFR $(-8 \mathrm{ml} / \mathrm{min} /$ year). In a study cohort of 26 patients treated for 24 months with agalsidase $\beta$, Breunig et al. [7] found GFR reductions of 6.5 (GFR $>90 \mathrm{ml} / \mathrm{min}$ at baseline) and 5.5 $\mathrm{ml} / \mathrm{min} /$ year (GFR $<90 \mathrm{ml} / \mathrm{min}$ at baseline). However, they excluded 3 patients with severe and progressive nephropathy necessitating dialysis. In the same study, there was a reduction in GFR of approximately $7.0 \mathrm{ml} / \mathrm{min} /$ year in female patients. Taken together, these studies show considerable variability in the reduction of GFR in patients receiving ERT with either agalsidase $\alpha$ or $\beta$. How- 
ever, the studies were carried out in small groups of patients and for a limited time period.

In contrast, the present analysis was conducted on data from a large number of patients $(n=165)$ receiving ERT for 3 years. The mean increase in serum creatinine and the mean reduction in eGFR in these patients were satisfactory compared with other reports. In men, there was a mean decrease of $2.66 \mathrm{ml} / \mathrm{min} / 1.73 \mathrm{~m}^{2} /$ year and in women of $1.20 \mathrm{ml} / \mathrm{min} / 1.73 \mathrm{~m}^{2} /$ year; in the total population, the mean decrease was $2.22 \mathrm{ml} / \mathrm{min} / 1.73 \mathrm{~m}^{2} /$ year. When patients were subdivided according to disease stage, reductions in eGFR were mostly similar to or lower than those reported in the literature (table 5). Thus, we have demonstrated substantial stabilization of renal function in all groups of patients with Fabry disease. Importantly, renal function remained stable throughout the 3 years of ERT in patients with severe renal failure (stage III).

Factors other than ERT cannot be ruled out when explaining the reduction in deterioration of renal function in treated patients with Fabry disease. ACE inhibitors and ARBs are used extensively in patients with Fabry disease and are effective in controlling blood pressure and providing renoprotection due to their well-known actions on glomerular hemodynamics and their interactions with cytokines [19-21].

The degree of proteinuria also critically affects the progression of Fabry nephropathy. Proteinuria $>1$ g/24 h is associated with a reduced response to ERT and a more rapid decline in renal function [22-24]. The patients analyzed in FOS had mild proteinuria (mean $<1 \mathrm{~g} / 24 \mathrm{~h}$ ) at baseline, which did not change during follow-up. Previously published data have also shown that ERT with either agalsidase $\alpha$ or $\beta$ does not alter urinary protein excretion $[25,26]$. Moreover, a sustained reduction in proteinuria associated with stabilization of renal function has been described in a small group of Fabry patients receiving ACE inhibitors and/or ARBs, with no significant change after addition of agalsidase $\beta$ therapy [27]. The use of ACE inhibitors and/or ARBs is therefore advisable to help prevent the deterioration in renal function and to reduce proteinuria in patients with Fabry disease, as it is for other proteinuric nephropathies $[28,29]$.

The present study has some limitations common to large multicenter, multinational studies: incomplete data reporting, differences in laboratory methods and the fact that there was no placebo group. In addition, the analysis focused only on patients with stage I-III renal disease and excluded patients with end-stage renal disease and renal transplant recipients. At present, controversy exists regarding the MDRD formula as a gold standard for estimating GFR, and therefore other formulae have been proposed [30]. The MDRD formula, however, remains a useful tool for investigating changes in renal function over time [31]. Despite the limitations common to outcome surveys, FOS provides consistent data indicating trends in renal function in patients with Fabry nephropathy on ERT.

Although we should be cautious about drawing definitive conclusions, the FOS data - obtained in normal clinical practice from a wide range of patients from many different countries - suggest that agalsidase $\alpha$ plays a significant role in slowing the progression of renal dysfunction in patients with Fabry nephropathy.

\section{Acknowledgments}

All authors are participants of the FOS, which is supported by Shire Human Genetic Therapies. We are indebted to Dr. David Campbell (Communigen, Oxford, UK) for his technical assistance in manuscript drafting.

\section{References}

1 Brady RO, Gal AE, Bradley RM, Martensonn E, Warshaw AL, Laster L: Enzymatic defect in Fabry's disease. Ceramide trihexosidase deficiency. N Engl J Med 1967;276:11631167.

- 2 Branton MH, Schiffmann R, Sabnis SG, Murray GJ, Quirk JM, Altarescu G, Goldfarb L, Brady RO, Balow JE, Austin HA III, Kopp JB: Natural history of Fabry renal disease: influence of alfa-galactosidase A activity and genetic mutations on clinical course. Medicine (Baltimore) 2002;81:122-138.
3 MacDermot KD, Holmes A, Miners AH: Anderson-Fabry disease: clinical manifestations and impact of disease in a cohort of 60 obligate carrier females. J Med Gen 2001;38: 769-775.

-4 Eng CM, Guffon N, Wilcox WR, Germain DP, Lee P, Waldek S, Caplan L, Linthorst GE, Desnick RJ: Safety and efficacy of recombinant human alfa-galactosidase A replacement therapy in Fabry's disease. $\mathrm{N}$ Engl J Med 2001;345:9-16
5 Schiffmann R, Kopp JB, Austin HA III, Sabnis S, Moore DF, Weibel T, Balow JE, Brady RO: Enzyme replacement therapy in Fabry disease: a randomized controlled trial. JAMA 2001;285:2743-2749.

-6 Lidove O, Joly D, Barbey F, Bekri S, Alexandra JF, Peigne V, Jaussaud R, Papo T: Clinical results of enzyme replacement therapy in Fabry disease: a comprehensive review of literature. Int J Clin Pract 2007;2:293-302. 
7 Breunig F, Weidemann F, Strotmann J, Knoll A, Wanner C: Clinical benefit of enzyme replacement therapy in Fabry disease. Kidney Int 2006;69:1216-1221.

$\checkmark 8$ Mehta A, Ricci R, Widmer U, Dehout F, Garcia de Lorenzo A, Kampmann C, Linhart A, Sunder-Plassmann G, Ries R, Beck M: Fabry disease defined: baseline clinical manifestations of 366 patients in the Fabry Outcome Survey. Eur J Clin Invest 2004;34:236-242.

$>9$ Peake M, Whiting M: Measurement of serum creatinine - current status and future goals. Clin Biochem Rev 2006;27:173-184.

$>10$ Levey AS, Bosch JP, Lewis JB, Greene T, Rogers N, Roth D: A more accurate method to estimate glomerular filtration rate from serum creatinine: a new prediction equation. Modification of Diet in Renal Disease Study Group. Ann Intern Med 1999;130:461-470.

- 11 Thurberg BL, Rennke H, Colvin RB, Dikman S, Gordon RE, Collins AB, Desnick RJ, O'Callaghan M: Globotriaosylceramide accumulation in Fabry kidney is cleared from multiple cell types after enzyme replacement therapy. Kidney Int 2002;62:1933-1946.

-12 Sakuraba H, Murata-Ohsawa M, Kawashima I, Taijma Y, Kotani M, Ohshima T, Chiba Y, Takashiba M, Jigami Y, Fukushige T, Kanzaki T, Itoh K: Comparison of the effects of agalsidase alfa and agalsidase beta on cultured human Fabry fibroblasts and Fabry mice. J Hum Genet 2006;51:180-188.

13 Schwarting A, Dehout F, Feriozzi S, Beck M, Metha A: Enzyme replacement therapy and renal function in 201 patients with Fabry disease. Clin Nephrol 2006;2:77-84.

14 Banikazemi M, Bultas J, Waldek S, Wilcox WR, Whitley CB, McDonald M, Finkel R, Packman S, Bichet DG, Warnock DG, Desnick RJ: Agalsidase beta therapy for advanced Fabry disease. Ann Intern Med 2007;146: 77-86.
15 De Schoenmakere G, Chauveau FD, Grunfeld JP: Enzyme replacement therapy in Anderson-Fabry's disease: beneficial effect on vital organ function. Nephrol Dial Transplant 2003;18:33-35.

16 Germain DP, Waldek S, Banikazemi M, Bushinsky D, Charrow J, Desnick RJ, Lee P, Loew T, Vedder AC, Abichandani R, Wilcox W, Guffon N: Sustained, long-term renal stabilization after 54 months of agalsidase $\beta$ therapy in patients with Fabry disease. J Am Soc Nephrol 2007;18:1547-1557.

17 Schiffmann R, Ries M, Timmons M, Flaherty JT, Brady RO: Long-term therapy with agalsidase alfa for Fabry disease: safety and effects on renal function in a home infusion setting. Nephrol Dial Transplant 2006;21: 345-354.

18 Schiffmann R, Askari H, Timmons M, Robinson C, Benko W, Brady RO, Ries M: Weekly enzyme replacement therapy may slow decline of renal function in patients with Fabry disease who are on long-term biweekly dosing. J Am Soc Nephrol 2007;18:1576-1583.

19 Wright JT, Bakris G, Greene T, Agodoa LY, Appel LJ, Charleston J, Cheek D, DouglasBaltimore JG, Gassman J, Glassock R, Herbert L, Jamerson K, Lewis J, Phillips RA, Toto RD, Middleton JP, Rostand SG; African American Study of Kidney Disease and Hypertension Study Group: Effect of blood pressure lowering and antihypertensive drug class on progression of hypertensive kidney disease: results from the AASK Trial. JAMA 2002;288:2421-2431.

20 Remuzzi G, Ruggenenti P, Perico N: Chronic renal diseases: renoprotective benefits of renin-angiotensin system inhibition. Ann Intern Med 2002;136:604.

21 Rule AD, Larson TS, Bergstralh EJ, Slezak JM, Jacobsen SJ, Cosio FG: Using serum creatinine to estimate glomerular filtration rate: accuracy in good health and in chronic kidney disease. Ann Intern Med 2004;12: 929-937.
22 Wilcox WR, Banikazemi M, Guffon N, Waldek S, Lee P, Linthorst GE, Desnick RJ, Germain GP: Long-term safety and efficacy of enzyme replacement therapy for Fabry disease. Am J Hum Genet 2004;75:65-74.

23 Warnock DG: Fabry disease: diagnosis and management, with emphasis on the renal manifestations. Curr Opin Nephrol Hypertens 2005;14:87-95.

-24 Breunig F, Wanner C: Update on Fabry disease: kidney involvement, renal progression and enzyme replacement therapy. J Nephrol 2008;21:32-37.

25 Warnock D: Enzyme replacement therapy and Fabry disease: quo vadis? J Am Soc Nephrol 2007;18:1368-1370.

26 Wanner C, Breunig F: Fabry nephropathy and the case for adjunctive renal therapy. J Am Soc Nephrol 2007; 18:2426-2428.

27 Tahir H, Jackson LL, Warnock DG: Antiproteinuric therapy and Fabry nephropathy: sustained reduction of proteinuria in patients receiving enzyme replacement therapy with agalsidase-beta. J Am Soc Nephrol 2007;18:2609-2617.

-28 Ruggenenti P, Perna A, Gherardi G, Benini $\mathrm{R}$, Remuzzi G: Chronic proteinuric nephropathies: outcomes and response to treatment in a prospective cohort of 352 patients with different patterns of renal injury. Am J Kidney Dis 2000;35:1155-1165.

29 Sarafidis PA, Khosla N, Bakris GL: Antihypertensive therapy in the presence of proteinuria. Am J Kidney Dis 2007;49:12-26.

-30 Stevens LA, Coresh J, Greene T, Levey AS: Assessing kidney function - measured and estimated glomerular filtration rate. $\mathrm{N}$ Engl J Med 2006;8:2473-2483.

-31 Sarnak MJ, Greene T, Wang X, Beck G, Kusek JW, Collins AJ, Levey AS: The effect of a lower target blood pressure on the progression of kidney disease: long-term follow-up of the Modification of Diet in Renal Disease Study. Ann Intern Med 2005;142:342-351.

\section{Erratum}

In the above article by S. Feriozzi et al. entitled 'Agalsidase alfa slows the decline in renal function in patients with Fabry disease' [Am J Nephrol 2009;29:353-361], in table $3 \mathrm{c}$ in the group of patients with protein $<500 \mathrm{mg} / 24 \mathrm{~h}$ (stage I), the value of mean change in eGFR of -2.10 is not correct and should read -12.42 . 